\title{
Risk Management in Sanità Il problema degli errori
}

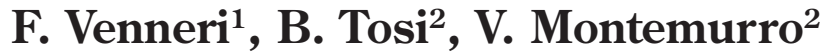 \\ ${ }^{1}$ Clinical Risk Manager ASL 10 Firenze \\ ${ }^{2}$ Reparto Dialisi, Ulivella e Glicini - IFCA - GIOMI
}

\section{Introduzione}

Sin dai primi anni Novanta, le problematiche legate all'errore in medicina, valutando, prima fra tutti, il numero degli errori svolti in ambito clinico, sono state ampiamente analizzate e studiate.

Partendo quindi, dalla valutazione numerica degli errori si è cercato di analizzarli nel loro divenire e di studiare il sistema al fine di prevenire altri sbagli.

Partendo da questi presupposti esistono due modalità di approccio al problema del rischio clinico:

- la prima si focalizza sul comportamento umano come fonte di errore agendo migliorando la conoscenza e la formazione individuale;

- la seconda si focalizza sulle condizioni nelle quali accade l'errore intese come l'insieme di elementi umani, tecnologici e relazionali, cercando di far emergere le situazioni che possono portare a sbagliare e rivedendone i processi di esecuzione, un approccio cosiddetto sistemico.

\section{Definizione di rischio clinico e di errore}

Il rischio clinico è la probabilità che un paziente sia vittima di un evento avverso, cioè subisca un qualsiasi "danno o disagio imputabile, anche in modo involontario, alle cure mediche prestate durante il periodo di degenza, che causa un prolungamento del periodo di degenza, un peggioramento delle condizioni di salute o la morte" (Kohn, IOM 1999).

Per evento avverso si intende un evento inatteso correlato al processo assistenziale e che comporta un danno al paziente, non intenzionale e indesiderabile. Gli eventi avversi possono essere prevenibili o non prevenibili. Un evento avverso attribuibile ad un errore è un evento avverso prevenibile.
Un evento sentinella è un evento avverso di particolare gravità, potenzialmente indicativo di un serio malfunzionamento del sistema, che può comportare morte o grave danno al paziente e che determina una perdita di fiducia dei cittadini nei confronti del servizio sanitario (1).

Gli errori organizzativi sono quelli determinati dalla strutturazione del sistema stesso, spesso dati dalla forte spinta del risparmio economico.

Fra questi basta pensare alla carenza d'organico sia medico che infermieristico che spesso affligge i nostri reparti. A tal proposito lo studio eseguito negli USA (2) per una valutazione corretta del numero di infermieri per degenti, ci può far capire come questa tipologia di errore sia abbastanza pressante nel nostro mondo.

Fra gli errori non secondo le intenzioni riconosciamo gli slips, che sono errori attenzionali e i lapses, che sono fallimenti della memoria.

Fra gli errori secondo le intenzioni, invece, riconosciamo i mistakes e le violazioni.

I mistakes sono errori pregressi che si sviluppano durante i processi di pianificazione e si dividono in ruled based (si applica una regola sbagliata per un errata percezione della situazione o si sbaglia nell'applicare una regola) e in knowledge based (errati percorsi ideativi o errata conclusione per deficit di conoscenza del soggetto).

Le violazioni sono, invece, delle azioni che deviano dalle procedure, dalle norme di sicurezza, dagli standard o dalle regole. Quelle di routine sono quelle scorciatoie che vengono prese ogni volta questo sia possibile; quelle eccezionali sono delle varianti che il soggetto mette in opera quando la situazione gli appare particolare (Tab. I).

Gli errori latenti sono associati ad attività distanti sia nel tempo che nello spazio, dal luogo dellincidente; per tale motivo le loro conseguenze possono restare silenti nel tempo e diventare evidenti solo quando si combinano ad altri rompendo in questo modo le difese del sistema stesso. 


\begin{tabular}{|c|c|c|}
\hline \multirow[t]{2}{*}{ AZIONI NON SECONDO LE INTENZIONI } & SLIP & $\begin{array}{l}\text { ERRORI ATTENZIONALI: } \\
\text { Intrusioni } \\
\text { Omissioni } \\
\text { Inversioni } \\
\text { Ordinamenti sbagliati } \\
\text { Tempi sbagliati }\end{array}$ \\
\hline & LAPSE & $\begin{array}{l}\text { ERRORI DELLA MEMORIA: } \\
\text { Omissione di elementi pianificati } \\
\text { Confusioni spaziali } \\
\text { Oblio delle intenzioni }\end{array}$ \\
\hline \multirow{2}{*}{ AZIONI SECONDO LA INTENZIONI } & MISTAKE & $\begin{array}{l}\text { RULED-BASED } \\
\text { Applicazione erronea di buone regole } \\
\text { Applicazione di regole sbagliate } \\
\text { KNOWLEDGE-BASED } \\
\text { Errori da deficit di conoscenza }\end{array}$ \\
\hline & VIOLAZIONI & $\begin{array}{l}\text { Violazioni di routine } \\
\text { Violazioni eccezionali } \\
\text { Atti di sabotaggi }\end{array}$ \\
\hline
\end{tabular}

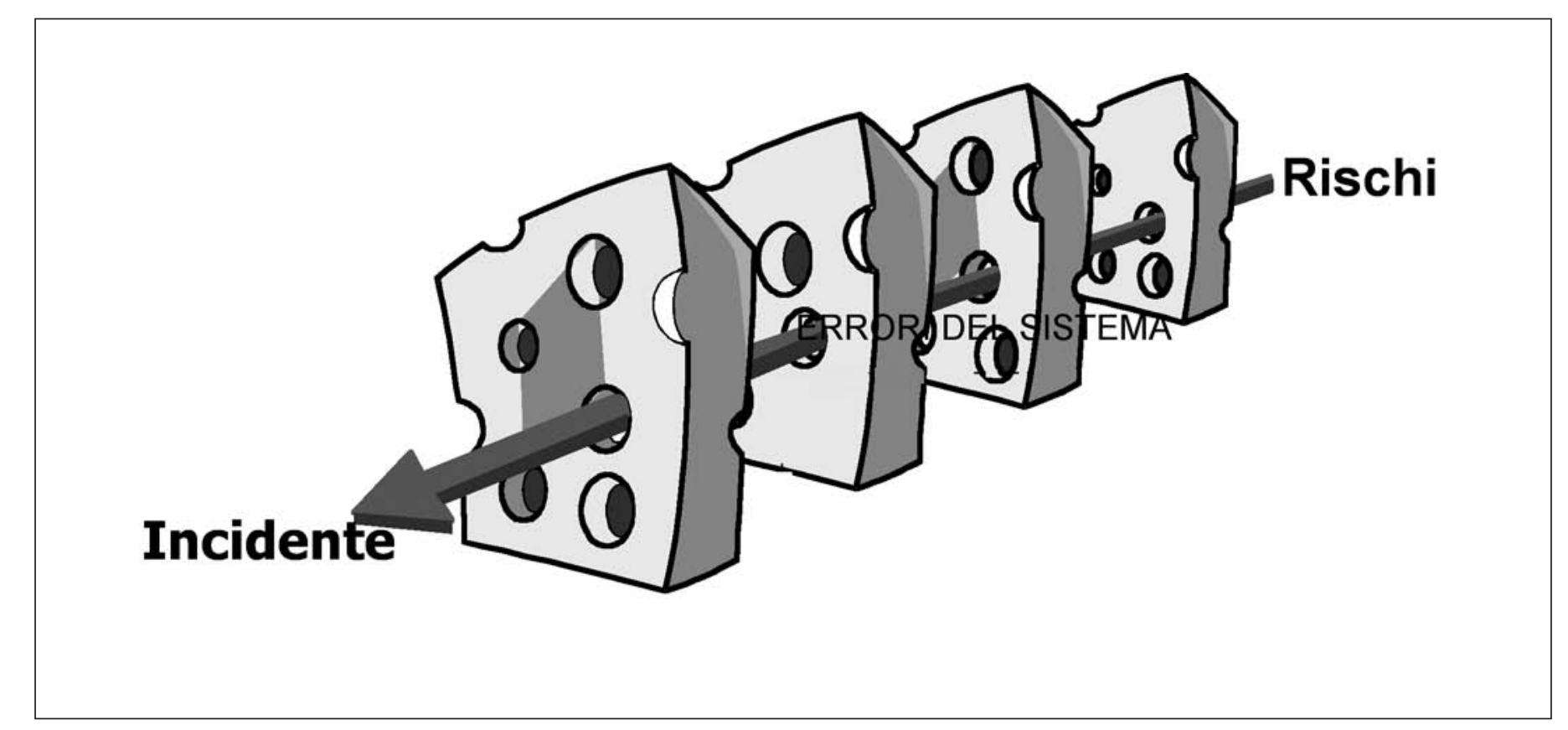

Fig. 1

Gli errori attivi, invece sono associati alle azioni degli operatori di prima linea, i loro effetti sono immediatamente percepiti e dunque facilmente individuabili.

L'incidente è spesso il frutto di una concatenazione di errori che insieme hanno superato tutte le difese del sistema (teoria del formaggio svizzero) (Fig. 1). Nella teoria del formaggio svizzero, ogni fetta di formaggio rappresenta una barriera del sistema: formazione, organizzazione, tecnologie, contesto operativo ecc. 


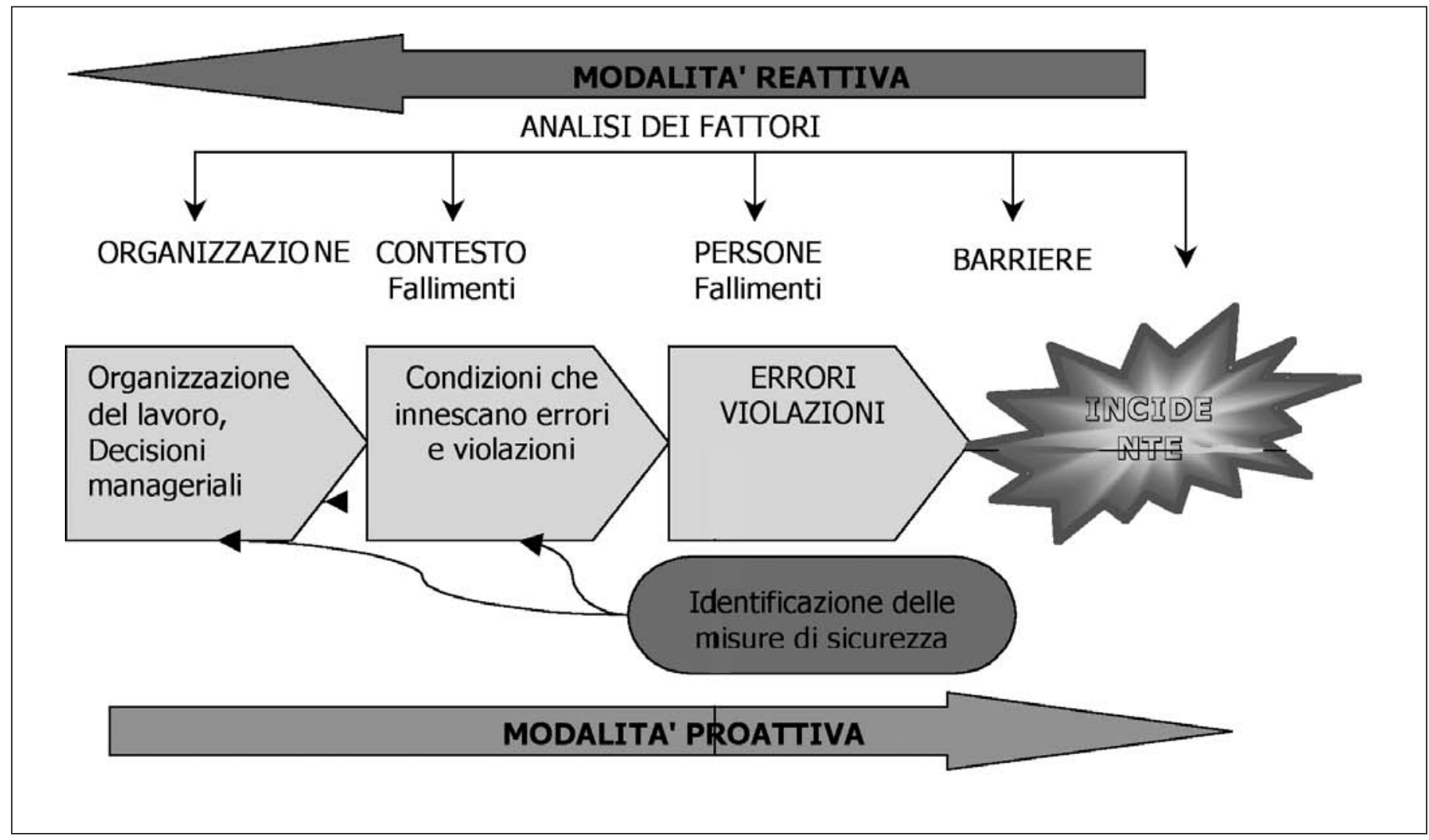

Fig. 2

\section{Metodi di analisi del rischio clinico}

Esistono due tipologie di analisi del rischio, un approccio detto proattivo e quindi proiettata a prevedere le ipotetiche fonti di errore, ed uno di tipo reattivo, proiettata alla valutazione a posteriori dell'errore stesso al fine che questo non si ripeta (Fig. 2).

Entrambe le due tipologie di analisi devono integrarsi, in un sistema complesso come quello sanitario, al fine di analizzare le fonti di errore su più versanti.

L'analisi proattiva (3) prevede lo studio di eventuali fonti di rischio clinico con interventi dediti a prevenire errori o complicanze in ambito sanitario.

Una parte di tale analisi prevede l'ergonomia cioè lo studio dell'usabilità delle apparecchiature, ed anche la costituzione e continua implementazione delle linee guida.

L'analisi reattiva coinvolge, invece l'operatore di prima linea che deve essere stimolato a comunicare gli eventuali eventi avversi o meglio i quasi eventi (near misses); a fronte, infatti, di un evento senti- nella, sono molto più numerosi i quasi errori, la cui analisi può aiutare a modificare il sistema al fine che si riducano i near misses e ancor più gli eventi sentinella.

\section{Conclusioni}

La problematica dell'errore in medicina deve coinvolgere tutti noi. Far meglio il nostro lavoro vuol dire dedicare al paziente sì, le cure necessarie, ma anche far sì che le nostre conoscenze e la nostra cultura possano dargli le migliori condizioni di cura.

Per far questo è importante sia l'aggiornamento clinico, sia una continua evoluzione della nostra figura professionale, apprendendo anche dagli errori sia nostri che di altri, ma soprattutto, dal punto di vista etico, adoperarsi al fine di ridurre le possibilità di sbagliare.

Quindi, mentre le strutture politiche ed amministrative cercano di creare una rete organizzativa per la gestione del rischio clinico, ogni figura professionale che dedica il suo lavoro in sanità dovrebbe adoperarsi nel suo piccolo a migliorare il mondo in cui lavora.

Anche solo la sensibilizzazione alla problematica e la segnalazione di eventi che hanno quasi portato 
all' errore può permettere tale miglioramento.

Ogni uomo che lavora riflettendo su come migliorare se stesso e perché no, anche gli altri, può far sì che il sistema stesso si presenti meno bucato (vedi formaggio svizzero).

Nel prossimo appuntamento saranno illustrati brevemente le metodologie di analisi degli eventi avversi in sanità: l'audit clinico GRC, la rassegna di morbilità e mortalità (M\&Ms) e la Root Cause Analysis (RCA).

\section{Indirizzo degli Autori:}

Francesco Venneri, MD

Via Serchio 40

59100 Prato

francesco.venneri@asf.toscana.it

\section{Bibliografia}

1. Ministero della Salute, Sicurezza dei pazienti e gestione del rischio clinico: Manuale per la formazione degli operatori sanitari. Disponibile su: http://www.ministerosalute. it/imgs/C_17_pubblicazioni_640_allegato.pdf

2. Baragatti L, Tonelli L. Quanti degenti per infermieri. Sanità, Il Sole 24 Ore, 26 febbraio-3marzo 2008.

3. Gaggiotti E, Semplici J. Sicurezza, qualità e risparmio in nefrologia. Obiettivo realizzabile?. Ed. Bios; 2004; 39-57; 101-

4. Reason JT. Managing the Risks of Organizational Accidents, 1997.

5. Reason JT. Human Error: Causes and Consequences, 1990.

6. Vincent C. Clinical Risk Management: Enhancing Patient Safety, 2001. 